Imperial/TP/97-98/072

TAUP-2517-98

hep-th/9809032

\title{
Free energy of $\mathcal{N}=4$ super Yang-Mills in Higgs phase and non-extremal D3-brane interactions
}

\author{
A.A. Tseytlin ${ }^{1,2 \star \dagger}$ and S. Yankielowicz ${ }^{3 *}$ \\ ${ }^{1}$ Theoretical Physics Group, Blackett Laboratory \\ Imperial College, London SWr 2BZ, U.K. \\ ${ }^{2}$ Department of Physics and Astronomy \\ Rutgers University, Piscataway NJ 08854-8019, USA \\ ${ }^{3}$ School of Physics and Astronomy \\ Beverly and Raymond-Sackler Faculty of Exact Sciences \\ Tel Aviv University, Ramat Aviv, 69978, Israel
}

\begin{abstract}
We study the free energy of $\mathcal{N}=4$ super Yang-Mills theory in the Higgs phase with a mass scale $M$ corresponding to non-zero v.e.v. of the scalar fields. At zero temperature this theory describes a system of parallel separated extremal D3-branes. Non-zero temperature corresponds to non-extremality in supergravity description. We interpret the supergravity interaction potential between a non-extremal D3-brane and a D3-brane probe as contribution of massive states to the free energy of the $N \rightarrow \infty$ SYM theory at strong 't Hooft coupling $\left(\lambda=2 N g_{\mathrm{YM}}^{2} \gg 1\right)$. Both low $(M \gg T)$ and high $(T \gg M)$ temperature regimes are considered. For low temperature we find that the structure of terms that appear in the free energy at strong and weak coupling is the same. The analysis of the high-temperature regime depends on a careful identification of the scalar field v.e.v. in terms of the distance between branes in the supergravity description and again predicts strong coupling terms similar to those found in the weak-coupling $\mathcal{N}=4$ SYM theory. We consider also the corrections to the strong coupling results by taking into account the leading $\alpha^{\prime 3} R^{4}$ string contribution to the supergravity effective action. This gives rise to the $\lambda^{-3 / 2}$ corrections in the coefficient functions of $\lambda$ which multiply different terms in free energy.
\end{abstract}

September 1998

\footnotetext{
* e-mail address: tseytlin@ic.ac.uk

$\dagger$ Also at Lebedev Physics Institute, Moscow.

* e-mail address: shimonya@post.tau.ac.il
} 


\section{Introduction}

D-branes, having both closed string and open string descriptions [1],2] provide an important link between certain physical quantities in supergravity and super Yang-Mills theory (see, in particular, [3, 4, 5, 6, 0, 8, 9]). While $N$ extremal D3-branes are described by a vacuum state of $\mathcal{N}=4 \mathrm{SYM}$ theory, the near-extremal D3-branes are expected to be described by a thermal state of the SYM theory.

The interpretation of near-extremal supergravity D-brane solutions as corresponding to finite temperature states on SYM side is very natural in view of the existence of the Hawking temperature and entropy associated with them. Indeed, the Bekenstein-Hawking entropy of non-extremal D3-brane has the same form as the entropy of the conformal SYM theory [3]. The relative coefficient of $3 / 4$ between the weakly coupled SYM result and the B-H entropy [3] reflects the need to interpolate from strong to weak coupling in order to compare the supergravity result to the perturbative SYM expression for the entropy [10].

Here we would like to address the question of a similar relation between the SYM thermodynamics in the massive Higgs phase (or Coulomb branch corresponding to the case of non-zero vacuum expectation values of the scalar fields) and the supergravity description of non-extremal D3-branes. As in [10] the aim will be to interpret the supergravity results as predictions about SYM free energy in the strong coupling regime.

Having non-zero temperature $T$ and non-zero scalar expectation values $U \sim M$ in SYM theory should correspond to a system of separated non-extremal D3-branes. We shall consider the simplest possible case when $N \gg 1$ coinciding D3-branes ('source') are separated by a distance (proportional to a mass scale $M$ ) from a single-charge D3-brane ('probe'). We shall assume that the parallel branes are separated along the single transverse direction, i.e. the $U(N+1)$ scalar (coordinate) matrix is $\hat{U}_{n}=\operatorname{diag}\left(\delta_{n 1} U, 0, \ldots, 0\right)$, breaking the gauge symmetry $U(N+1) \rightarrow U(N) \times U(1)$. We shall be interested in the case when the whole system is in a thermal state with temperature $T$ proportional to the non-extremality parameter of the D3-brane solution. The aim will be to compute the supergravity interaction potentiali and to interpret it as a contribution of massive states to the free energy of the $N \rightarrow \infty$ SYM theory at strong 't Hooft coupling $\left(\lambda=2 N g_{\mathrm{YM}}^{2} \gg 1\right)$.

At zero temperature the static interaction potential between the source and the probe vanishes - parallel branes represent a supersymmetric BPS state. At finite temperature it is natural to expect that there will be an attractive potential between the branes, i.e. there will be a tendency to restore the broken gauge symmetry.

This expectation is in agreement with the fact that the extremal D3-brane solution [14,15] with separated centers does not have a non-extremal generalisation which is static

1 Some previous related discussions appeared in 6, 11, 12, 13. 
- the only static non-extremal solution is a single-center black D3-brane [14]. In general, non-extremal black holes attract and fall onto each other, i.e. one obtains a static solution only if they are at the same point (we exclude the possibility of having an infinite periodic array of black holes). Extremal BPS black holes or branes form a much larger space of static solutions parametrised by harmonic functions, i.e. they can be separated and put at different points at no const in energy.

This is what happens in gauge theory at weak coupling: if we start with a vacuum state with a non-zero scalar value $U \sim M$ and switch on the temperature $T \gg M$, the induced scalar potential $a_{1} T^{4}+a_{2} T^{2} U^{2}+\ldots$ will drive the system back to the unbroken phase with $\langle U\rangle=0$ [16]. The finite temperature breaks supersymmetry and lifts the vacuum degeneracy of $\mathcal{N}=4 \mathrm{SYM}$ theory. This is seen at weak coupling, and we interpret the above remark about supergravity solutions as the prediction that the same happens also at large $N$ and strong 't Hooft coupling.

Below we shall consider the supergravity expression for the interaction potential between non-extremal D3-brane 'source' and D3-brane 'probe'. We shall interpret it in terms of the massive states contributions to the SYM free energy $F$ as a function of the temperature $T$ and mass scale $M \sim U$ (or separation between branes) in Higgs phase at strong coupling, and also compare it with the weak-coupling SYM theory result for the free energy. We shall find that while the weak and strong coupling expressions contain terms of similar structure, their coefficients are, in general, different. This follows the same pattern as discovered in [3, 10] for the coefficient in the free energy $\sim V_{3} T^{4}$ in the massless (coinciding brane) case. As in [17,10 we shall consider the modification of the supergravity expressions by the leading $\alpha^{\prime 3} R^{4}$ string correction and suggest (and present evidence) that at finite 't Hooft coupling $\lambda$ different terms in $F(T, M)$ are multiplied by non-trivial functions of $\lambda$, which at strong coupling go as $a+b \lambda^{-3 / 2}+\ldots$.

We shall mostly concentrate on the case when separation is large compared to nonextremality, i.e. when the temperature is much smaller than the mass scale. When $M \gg$ $T$ the contribution of the massive states to the SYM free energy we are interested in may be computed in two steps. One may first ignore the temperature and integrate (in the $N \rightarrow \infty$ limit) the massive modes out obtaining a Wilsonian effective action $(W \sim$ $\left.\int d^{4} x \sum \lambda^{l-1} \frac{F^{2 l+2}}{M^{4 l}}\right)$ for the massless modes. One may then switch on the temperature and compute the free energy starting with this effective action. On the supergravity side, the probe's action in an extremal source background is known to be related to $W$. Given that the structure of the static potential part of the probe's action in a non-extremal

2 We are assuming that there is no phase transition at finite $\lambda$ (for some arguments to the opposite see [18]). 
background is very similar to that of the velocity-dependent part in the extremal case, it may not be completely surprising that one finds also a close correspondence between the thermal average of $W$ and the interaction potential of a D3-brane probe with a nonextremal D3-brane source.

In section 2 we shall compute the supergravity interaction potential as a function of non-extremality parameter $\left(r_{0} \sim T\right)$ and separation $(r \sim M)$. We shall consider the large charge (or 'near-core') approximation.

In section 3 we shall study the 'low-temperature' or 'long-distance' $(M \gg T)$ expansion of the resulting free energy and compare the first two leading terms $\left(c_{1} T^{4}+c_{2} \frac{T^{8}}{M^{4}}\right)$ with the corresponding terms in the weak-coupling SYM free energy. The $\frac{T^{8}}{M^{4}}$ term will be shown to a have a 3-loop origin in the SYM perturbative expansion. In general, the $\frac{T^{4 l+4}}{M^{4 l}}$ term in $F$ has $(l+2)$-loop origin in the SYM free energy and is related to the term $\frac{F^{2 l+2}}{M^{4 l}}$ in the effective Wilsonian action at $T=0$.

In section 4 we shall discuss the opposite 'high-temperature' or 'short-distance' ( $T \gg$ $M)$ expansion. The comparison of this expansion with the corresponding SYM expansion a priori may seem less reliable. Unlike the previous case where the strings between the source and probe are long and, therefore, represent very massive states, in the present case the temperature fluctuations are much larger then the Higgs scale (or separation between branes). Nevertheless, in view of the successful understanding of the thermodynamics of coinciding branes, it would be interesting to see how much of the physics in this regime is accounted for by the supergravity expansion. As explained in Appendix, while the $M$-dependent part of the 1-loop free energy vanishes exponentially in the $M \gg T$ limit, expanded for $T \gg M$ it contains the well-known $T^{2} M^{2}$, $T M^{3}$, etc., terms. Similar terms are found in the supergravity potential, suggesting that they should be present in the strong-coupling SYM free energy.

In section 5 we consider the $\lambda^{-3 / 2}$ corrections to the coefficients in the strong-coupling expression for the free energy produced by taking into account the leading $\alpha^{\prime 3} R^{4}$ string contribution to the supergravity action.

In Appendix we present the expression for the 1-loop effective potential in $\mathcal{N}=4$ SYM theory at finite temperature.

\section{Supergravity expression for D3-brane interaction potential}

We shall consider a D3-brane probe (with charge 1) in a non-extremal D3-brane background (with charge $N$ ) and find the static interaction potential as a function of nonextremality parameter $r_{0}$ and separation $r$. This potential will be attractive and will 
vanish at large separation (it will vanish also for $r_{0} \rightarrow 0$ corresponding to the BPS limit). It will be interpreted as a free energy of a $U(N+1)$ SYM gas at finite temperature (related to $r_{0}$ ) and non-zero scalar vacuum expectation value or mass scale $M$ (related to $r$ ).

The metric of the non-extremal D3-brane and the 'electric' components of the 4-form potential are $(i=1,2,3)$

$$
\begin{gathered}
d s^{2}=H^{-1 / 2}\left(-\mathrm{f} d t^{2}+d y_{i} d y_{i}\right)+H^{1 / 2}\left(\mathrm{f}^{-1} d r^{2}+r^{2} d \Omega_{5}^{2}\right) \\
C_{0123}=H^{-1}\left(1-H_{0}\right)
\end{gathered}
$$

where

$$
\begin{gathered}
H_{0}=1+\frac{L^{4}}{r^{4}}, \quad H=1+\frac{\tilde{L}^{4}}{r^{4}}, \quad \mathrm{f}=1-\frac{r_{0}^{4}}{r^{4}}, \\
L^{4}=4 \pi g_{s} N \alpha^{2}, \quad \tilde{L}^{4}=\sqrt{L^{8}+\frac{1}{4} r_{0}^{8}}-\frac{1}{2} r_{0}^{4} .
\end{gathered}
$$

The string coupling $g_{s}$ is related to the SYM coupling $g_{\mathrm{YM}}$ and the 't Hooft coupling $\lambda$ by

$$
g_{\mathrm{YM}}^{2}=2 \pi g_{s}, \quad \lambda \equiv 2 N g_{\mathrm{YM}}^{2}=4 \pi N g_{s}=L^{4} \alpha^{\prime-2}
$$

The non-extremality parameter is related to the Hawking temperature $T$ as follows [3, 19]

$$
r_{0}=\pi L^{2} T
$$

We shall consider D3-brane probe to be static and parallel to the source and choose the static gauge (that such configuration is not a classical solution without external force will not be important for us). We shall assume that the euclidean time (same for the probe and the source) has period $\beta=\frac{1}{T}$. Then the interaction part of the action for the D3-brane probe takes the form 3

$$
I=T_{3} V_{3} \beta H^{-1}\left(\sqrt{\mathrm{f}}-1+H_{0}-H\right),
$$

where $V_{3}$ is the volume of the spatial directions along the D3-brane and $T_{3}$ is the D3-brane tension [1]

$$
T_{3}=\frac{1}{2 \pi g_{s}\left(2 \pi \alpha^{\prime}\right)^{2}}=\frac{N}{2 \pi^{2} L^{4}}
$$

We shall assume that $N \rightarrow \infty, \lambda \gg 1$ for the validity of the supergravity description [4,5,6] and also consider the low-energy or gauge theory limit [4, 12,7] in which

$$
\alpha^{\prime} \rightarrow 0, \quad\left\{U=\frac{r}{\alpha^{\prime}}, \quad U_{0}=\frac{r_{0}}{\alpha^{\prime}}=\pi \lambda^{1 / 2} T, \quad g_{\mathrm{YM}}^{2}=2 \pi g_{s}, T\right\}=\text { fixed . }
$$

\footnotetext{
3 Similar actions were discussed in [6, 11, 12].
} 
Equivalently, one may set $\alpha^{\prime}=1$ but take $L \gg r, L \gg r_{0}$ (i.e. $\tilde{L} \rightarrow L$ ). In this limit

$$
H \rightarrow H_{0} \rightarrow \frac{L^{4}}{r^{4}}=\frac{\lambda}{U^{4}}, \quad \mathrm{f}=1-\frac{r_{0}^{4}}{r^{4}}=1-\frac{U_{0}^{4}}{U^{4}}
$$

The resulting metric [7] that follows from (2.1) has horizon at $U=U_{0}$ and a curvature singularity at $U=0[20]$. The finite temperature $\mathcal{N}=4$ SYM theory should describe the full geometry including the under-horizon region 20. 0 . The vielbein components of the Weyl tensor are proportional to $\frac{U_{0}^{4}}{\lambda^{1 / 2} U^{4}}$ and are small near (and outside) the horizon where one can thus trust the supergravity description.

The action of the probe placed outside of the horizon (2.6) now becomes

$$
I \equiv \beta F=\beta V_{3} T_{3} L^{-4} r^{4}\left[\sqrt{1-\frac{r_{0}^{4}}{r^{4}}}-1\right] .
$$

The corresponding 'free energy' is

$$
F=V_{3} \frac{N U^{4}}{2 \pi^{2} \lambda^{2}}\left[\sqrt{1-\frac{\pi^{4} \lambda^{2} T^{4}}{U^{4}}}-1\right]
$$

or, equivalently,

$$
F=\frac{1}{2 \pi^{2}} V_{3} N M^{4}\left[\sqrt{1-\frac{\pi^{4} T^{4}}{M^{4}}}-1\right], \quad M \equiv \frac{r}{L^{2}}=\frac{U}{\lambda^{1 / 2}} .
$$

$M=\frac{r}{L^{2}}$ and $T=\frac{r_{0}}{\pi L^{2}}$ are the natural parameters corresponding to the supergravity solution with the characteristic scale $L$. However, in the comparison with the SYM perturbation theory in section 3.2 the role of the mass parameter will be played by $U$. Note that the temperature mass scales in the SYM theory are $M_{0}=T$ (tree-level fermion mass), $M_{e l}=\lambda^{1 / 2} T$ (1-loop scalar mass and the 'electric' gauge boson mass) and $M_{m a g n}=\lambda T$ (magnetic screening scale at which finite $T$ theory becomes non-perturbative). $M_{e l}$ is associated with the non-analytic $M_{e l}^{3} T=\lambda^{3 / 2} T^{4}$ term in the free energy [21].

Below we will interpret the expression (2.11) as the $N \rightarrow \infty, \lambda \gg 1$ limit of the SYM free energy at temperature $T$ in the phase with a non-zero scalar expectation value $U$.

The supergravity potential (2.11) if interpreted as the exact expression for the SYM free energy in the strong-coupling limit suggests the existence of the maximal temperature (for given $U$ and $\lambda$ ). The square root formula (2.11) has the same 'Born-Infeld' origin (cf.(2.6)) as the action of a relativistic D-particle, so the bound on $T$ is similar to the

4 The gauge theory in a thermal state should represent the D3-brane in thermal equilibrium with a gas of string modes in $A d S_{5}$ [7, 20]. 
maximal velocity or maximal field strength bounds. Since (2.11) is formally valid in the low-temperature phase, this suggests that $T_{c}=\pi^{-1} \lambda^{-1 / 2} U$ may be a 'critical temperature' at which a phase transition takes place.5 Note that the entropy and heat capacity corresponding to the free energy (2.11)

$$
S=-\frac{\partial F}{\partial T}=\frac{N \pi^{2} V_{3} T^{3}}{\left(1-\frac{\pi^{4} \lambda^{2} T^{4}}{U^{4}}\right)^{1 / 2}}, \quad C=T \frac{\partial S}{\partial T}=\frac{3 N \pi^{2} V_{3} T^{2}\left(1-\frac{\pi^{4} \lambda^{2} T^{4}}{3 U^{4}}\right)}{\left(1-\frac{\pi^{4} \lambda^{2} T^{4}}{U^{4}}\right)^{3 / 2}}
$$

diverge at the critical temperature.

In the above expressions we did not yet make any assumptions about the relative values of $r$ and $r_{0}$ (or $U$ and $U_{0}$ ). As in [11,223,24] we shall think of the non-extremal D3brane as 'located' at the horizon. Thus the limit of large separation between the branes or 'small temperature' $M \gg T$ limit is $r \gg r_{0}$ (or $U \gg U_{0}$ ) while the limit of small separation or 'high temperature' $T \gg M$ is $r \simeq r_{0}\left(\right.$ or $\left.U \simeq U_{0}\right)$.

\section{Long-distance or low temperature expansion}

Let us first consider the long-distance approximation $r \gg r_{0}$ corresponding to the probe being far away from the source. Equivalently, let us assume that the temperature is very low, i.e. the scalar expectation value or the mass parameter $U$ is much larger than $U_{0}=\pi \lambda^{1 / 2} T$.

\subsection{Leading term}

The leading term in the long-distance or low-temperature expansion of (2.11) is

$$
F=-\frac{1}{2} T_{3} V_{3} L^{-4} r_{0}^{4}=-\frac{1}{4} N \pi^{2} V_{3} T^{4}
$$

Remarkably, this expression is perfectly consistent with the previous results [3, [10] about the correspondence between the entropy or free energy of the massless SYM gas and the Bekenstein-Hawking entropy of near-extremal D3-brane. The free energy of $U(\hat{N})$ SYM theory in the zero scalar (massless) vacuum is given (for $\hat{N} \gg 1$ ) by [10]

$$
\begin{gathered}
F=-\frac{1}{6} f(\lambda) \pi^{2} V_{3} \hat{N}^{2} T^{4}, \\
f(\lambda \rightarrow 0) \rightarrow 1, \quad f(\lambda \gg 1)=\frac{3}{4}+\frac{45}{32} \zeta(3) \lambda^{-3 / 2}+\ldots \rightarrow \frac{3}{4} .
\end{gathered}
$$

5 While there is no phase transition in the conformal case $(U=0)$ [22], it may be present in the Higgs phase. 
Taking

$$
\hat{N}=N+1
$$

one thus finds that in the strong coupling (supergravity) limit

$$
F=-\frac{1}{8}\left(N^{2}+2 N+1\right) \pi^{2} V_{3} T^{4} .
$$

In the weakly coupled SYM theory the separation of one of the D3-branes from $N$ others corresponds to switching on a non-zero scalar expectation value.6 Then the $O\left(N^{2}\right)$ and $O(1)$ terms in the total free energy (3.4) correspond to the contributions of the modes which remain massless while the $O(N)$ term is the contribution of the massive modes. The $O(N)$ term in (3.4) is exactly the expression in (3.1) which represents the energy of the separated brane. This is consistent with the interpretation (see, e.g., 250) of the probe's action as being a result of intergrating out the massive SYM modes (or strings connecting probe and source): the analogue of (3.1) at weak coupling is the contribution to the total free energy produced by the loop of massive modes.

In general, one could expect that the total large $N$ free energy of the SYM gas in the non-zero scalar (or 'one brane separated') phase may have the form

$$
F=-\frac{1}{6} \pi^{2} V_{3} T^{4}\left[f(\lambda) N^{2}+2 \tilde{f}(\lambda) N+\ldots\right] .
$$

What we have found is that the functions $f$ and $\tilde{f}$ have the same weak and strong coupling limits and thus are likely to coincide also for finite 't Hooft coupling. We shall present a non-trivial check of this conjecture in section 6 by showing that $f$ and $\tilde{f}$ do have the same $O\left(\lambda^{-3 / 2}\right)$ terms in their strong-coupling expansions.

\subsection{Subleading term}

The first subleading term in the large-distance expansion of (2.11) is

$$
F_{1}=-\frac{1}{16} V_{3} \pi^{6} N \lambda^{2} \frac{T^{8}}{U^{4}} .
$$

The supergravity predicts that this term should be present in the strong-coupling limit of the SYM theory free energy in the 'massive' phase. As we shall argue below, like in the $T^{4}$ term case, there is a similar $\frac{T^{8}}{U^{4}}$ term also in the weak-coupling expression for the SYM free energy. This term has a 3-loop origin.

6 Since $U^{2}=U_{n} U_{n} \quad(n=1, \ldots, 6)$ we may assume that branes are separated along $n=1$ direction, i.e. take the $U(N+1)$ scalar matrix as $U_{1}=\operatorname{diag}(U, 0, \ldots, 0)$. 
It is useful to draw analogy between the large-distance expansion of the non-extremal $(T \neq 0)$ static potential $(2.10),(2.11)$ and the velocity-dependent D3-brane interaction potential in the extremal caset

$$
\mathcal{V}=T_{3} V_{3} L^{-4} r^{4}\left[\sqrt{1-\frac{L^{4} v^{2}}{r^{4}}}-1\right]
$$

The leading $v^{2}$ term corresponds to the classical $U(1) \quad F_{\mu \nu}^{2}$ term on the SYM side, the $\frac{v^{4}}{r^{4}}$ term is reproduced by the 1-loop $\frac{F^{4}}{r^{4}}$ term, the $\frac{v^{6}}{r^{8}}$ term - by the 2-loop $\frac{F^{6}}{r^{8}}$ term, etc. (see, e.g., [2,26,27,28] and refs. there). The reason for the agreement between the supergravity expressions and the weak-coupling SYM results lies in the known [29] and expected non-renormalisation theorems of the relevant terms in the large $N$ SYM effective action: the perturbative $l$-loop terms $\left(N g_{\mathrm{YM}}^{2}\right)^{l-1} \frac{F^{2 l+2}}{M^{4 l}}$ should not be multiplied by functions of $\lambda=2 N g_{\mathrm{YM}}^{2}[27,30,28]$.

At the same time, no such non-renormalisation theorems are expected in the nonextremal or finite temperature case where supersymmetry is broken. As already found on the example of the $T^{4}$ term [10], different terms in the free energy may be multiplied by non-trivial functions of the SYM coupling, i.e. one may find the following expression for the 'massive' contribution to the free energy

$$
F(U \gg T)=-\frac{1}{3} \pi^{2} V_{3} N T^{4}\left[f(\lambda)+h_{1}(\lambda) \lambda^{2} \frac{T^{4}}{U^{4}}+h_{2}(\lambda) \lambda^{4} \frac{T^{8}}{U^{8}}+\ldots\right],
$$

where, like $f$ in (3.3), the functions $h_{n}$ approach finite values both at small and large $\lambda$, i.e.

$$
h_{n}(\lambda \rightarrow 0) \rightarrow a_{n}, \quad h_{n}(\lambda \gg 1) \rightarrow b_{n}+c_{n} \lambda^{-3 / 2}+\ldots .
$$

The coefficients $b_{n}$ are determined by the supergravity action (2.11).

Let us now argue that (3.8),(3.9) is consistent also with the weakly coupled SYM theory. In general, the perturbative (planar) loop expansion for the $\mathcal{N}=4 \mathrm{SYM}$ free energy as a function of temperature and scalar vacuum value or effective mass parameter $M=U$ has the following form (determined in view of the UV finiteness of the theory by dimensional considerations)

$$
F=V_{3} T^{4}\left[\mathcal{F}_{1}\left(\frac{T}{U}\right)+\lambda \mathcal{F}_{2}\left(\frac{T}{U}\right)+\lambda^{2} \mathcal{F}_{3}\left(\frac{T}{U}\right)+\ldots\right] .
$$

7 Note that as in (2.12) one can rescale the coordinates $\mathrm{r}=\frac{r}{L^{2}}, \mathrm{v}=\frac{v}{L^{2}}$, absorbing all powers of $L$, so that $\mathcal{V}=\frac{1}{2 \pi^{2}} N V_{3} \mathrm{r}^{4}\left[\sqrt{1-\frac{\mathrm{v}^{2}}{\mathrm{r}^{4}}}-1\right]$.

8 The field normalizations in the SYM Lagrangian are $L \sim \frac{1}{g_{\mathrm{YM}}^{2}} \operatorname{tr}\left(F^{2}+D U D U+U^{4}+\ldots\right)$. 
To compare with long-distance expansion in supergravity we are interested in the lowtemperature limit when $U \gg T$. Then it is easy to show (see Appendix) that the nonconstant $U$-dependent part of the 1-loop correction vanishes exponentially in this limit, $\mathcal{F}_{1}-\frac{\pi^{2}}{6} \rightarrow a \frac{U^{2}}{T^{2}} e^{-\sqrt{\pi} \frac{U}{T}} \rightarrow 0$. The same conclusion happens to apply also to $\mathcal{F}_{2}$. However, the 3-loop term $\mathcal{F}_{3}$ does contain a nontrivial contribution proportional to $\frac{T^{4}}{U^{4}}$ which thus has the same structure as the strong-coupling (supergravity) correction (3.6). This is in agreement with the ansatz (3.8), (3.9).

A simple way to argue that the 3-loop term in the SYM free energy contains a nontrivial $\frac{T^{8}}{U^{4}}$ contribution is the following. The 1-loop SYM effective action at $T=0$ is known to have the $N \frac{F^{4}}{U^{4}}$ term (its supersymmetric extension) in the low-energy, large $U$ expansion. Here $F$ is the $U(N)$ massless gauge field strength remaining after integrating out massive string modes ( $F^{4}$ stands for several terms with different Lorentz index contractions). The thermal averaging of $N \frac{F^{4}}{U^{4}}$ to the leading order in gauge coupling then leads to the required term (see also [31] for analogous discussion in the context of QED). Indeed,

$$
<F^{4}>=\left[c\left(N g_{\mathrm{YM}}^{2}\right)^{2}+O\left(\left(g_{\mathrm{YM}}^{2}\right)^{4}\right)\right] T^{8} .
$$

The resulting coupling dependence is as appropriate for a 3-loop planar graph contribution to the free energy (averaging is done with $e^{-F^{2} / g_{\mathrm{YM}}^{2}}$ measure). The $T^{8}$ behaviour follows on dimensional grounds. 9

Remarkably, the supergravity (strong-coupling) result in (3.6) $\frac{r_{0}^{8}}{r^{4}} \sim \frac{T^{8}}{U^{4}}$ also has the coefficient $\left(N g_{\mathrm{YM}}^{2}\right)^{2}$. Though we did not attempt compute the proportionality constant in the weak-coupling 3-loop SYM expression (3.11), there is no reason to expect that it will coincide with the corresponding constant in the supergravity expression (3.6): the weak-coupling and strong-coupling coefficients $a_{1}$ and $b_{1}$ in (3.9) are most likely different (as they are in $(3.3)$ ). We shall confirm the presence of the $\lambda^{-3 / 2}$ correction in the strongcoupling expansion of the function $h_{1}(\lambda)$ in $(3.9)$ in section 5 , suggesting, by analogy with the $T^{4}$ term [10], that there is, indeed, a non-trivial renormalisation of the coefficient of the $\lambda^{2} \frac{T^{8}}{U^{4}}$ term in the free energy.

Similar conclusions seem to apply to other terms in the low-temperature expansion of the free energy (3.8): the structure of the terms $T^{4}\left(\lambda^{2} \frac{T^{4}}{U^{4}}\right)^{l}$ is the same in the weak and

9 To demonstrate that the expectation value at $T \neq 0$ is proportional to $T^{8}$ it is enough to consider the abelian approximation. Separating first the points in $F^{4}$ as, e.g., $<F^{2}(x) F^{2}(0)>$ we get terms like $\partial \partial G \partial \partial G$, where $G$ is the massless scalar propagator at finite $T$, i.e. $G(x) \sim$ $\sum_{n}\left[|x|^{2}+\left(x_{0}-n T^{-1}\right)^{2}\right]^{-1}$. Differentiating and taking the limit of coinciding points we obtain the $T^{8}$ result (using that the UV cutoff dependence should cancel out as it is absent at $T=0$ ). 
strong coupling limits while their coefficients may be different. Again, to argue that such terms appear (at $2 l+1$ loop order) in the weak-coupling expression for free energy one may start with the fact that there is the $\lambda^{l-1} \frac{F^{2 l+2}}{U^{4 l}}$ term in the l-loop zero-temperature low-energy SYM effective action (which, as was mentioned above, should reproduce the $\frac{v^{2 l+2}}{r^{4 l}}$ term in (3.7)). Taking the expectation value of the $F^{2 l+2}$ operator at $T \neq 0$ and in the planar limit (that adds $l+1$ to the total loop order) one gets the required factor of $\lambda^{l+1} T^{4 l+4}$. Note that the presence of the non-trivial functions $h_{n}(\lambda)$ in (3.8) (i.e. a breakdown of non-renormalisation theorems) may be attributed to the second step of thermal averaging (cf.(3.11)).

\section{Short distance or high temperature expansion}

The perturbative free energy of the SYM theory with mass scale $M$ has the following high temperature or small mass $(T \gg M)$ expansion (see Appendix):

$$
F(T \gg M)=a_{1} T^{4}+a_{2} T^{2} M^{2}+a_{3} T M^{3}+\ldots
$$

i.e. the functions $\mathcal{F}_{n}$ in $(3.10)(M \sim U)$ are $\mathcal{F}_{n}(T \gg M)=k_{n}+p_{n} \frac{M^{2}}{T^{2}}+q_{n} \frac{M^{3}}{T^{3}}+\ldots$ To see if the supergravity expression (2.10) representing strong-coupling limit of the SYM free energy contains similar $T^{2} M^{2}$, etc., terms one is to consider its short-distance or nearhorizon $r \rightarrow r_{0}$ (or $U \rightarrow U_{0}$ ) expansion. The action (2.10) will be describing a D3-brane probe separated by a small distance from the horizon $U=U_{0}$ of a non-extremal D3-brane source.10

One immediate question is how to relate the supergravity coordinate $r$ in the region where it is close to $r_{0}$ to the scalar condensate or mass scale $M$ of SYM theory. The relation between the scalar field value $U \sim M$ and $r$ here appears to be more complicated than the direct proportionality $r \sim M$ which applies for large $r$ (cf. (2.8)).

The required identification is suggested by the proper choice of coordinates in the near-horizon region. As was noted in [11], to compare the supergravity description with the SYM theory one in the non-extremal case one should relate the SYM scalars not to $r$ but to the new 'isotropic' coordinate $\rho$ defined by

$$
\mathrm{f}^{-1} d r^{2}+r^{2} d \Omega_{5}^{2}=\chi(\rho)\left(d \rho^{2}+\rho^{2} d \Omega_{5}^{2}\right)
$$

10 One may wonder if the limit of small separation between branes should correspond to the expansion near $r=0$ where the brane source is supposed to be located in the extremal case. Note, however, that in the limit (2.8) or $L \gg r, r_{0}$ both $r$ and $r_{0}$ are effectively sent to zero. In any case, the supergravity description is valid for $U \geq U_{0}$ but is not valid for $U \rightarrow 0$. 


$$
\rho=\exp \int \frac{d r}{r \sqrt{f}}=\left(r^{2}+\sqrt{r^{4}-r_{0}^{4}}\right)^{1 / 2}, \quad r^{2}=\frac{\rho^{4}+r_{0}^{4}}{2 \rho^{2}} .
$$

Note that $\rho \rightarrow \rho_{0}=r_{0}$ when $r \rightarrow r_{0}$. Expressed in terms of $\rho$ the free energy in (2.10) becomes

$$
F=-\frac{1}{2} V_{3} T_{3} L^{-4} r_{0}^{4}\left(1+\frac{r_{0}^{4}}{\rho^{4}}\right) .
$$

This change of coordinates was not esential in the above discussion of the large distance expansion, 11 but is crucial for the correct SYM interpretation of the probe's action in the near-horizon region.

Expanding $\rho$ near $r_{0}$ and introducing the scalar mass parameter $M$ as (see (2.4),(2.5); cf.(2.12))

$$
M=\frac{\rho-r_{0}}{L^{2}}, \quad \rho-r_{0}=\alpha^{\prime} \lambda^{1 / 2} M, \quad \text { i.e. } \quad \rho=\alpha^{\prime} \pi \lambda^{1 / 2}\left(T+\pi^{-1} M\right),
$$

we find 12

$$
F=-\frac{1}{4} \pi^{2} V_{3} N T^{4}\left[1+\frac{1}{\left(1+\frac{M}{\pi T}\right)^{4}}\right] .
$$

For small $M$ or high $T$ (by 'high temperature' expansion we mean a near-horizon nearextremal expansion where both $M$ and $T$ are small but $T \gg M$ ) we get

$$
F=-\frac{1}{4} \pi^{2} N V_{3}\left(2 T^{4}-4 \pi^{-1} T^{3} M+10 \pi^{-2} T^{2} M^{2}-20 \pi^{-3} T M^{3}+\ldots\right)
$$

Let us discuss the meaning of the several leading terms in (4.7). The first $T^{4}$ term in (4.6) (whose coefficient does not actually depend on how we relate $r$ to $M$ at $r \rightarrow r_{0}$ ) is similar to the $r \rightarrow \infty$ result (3.1) but its coefficient is different by the factor of 2 . It has the following SYM interpretation. For small $r \sim r_{0}$ we have a $U(N+1)$ SYM theory describing all $N+1$ D3-branes close to each other. Taking large $r \gg r_{0}$ corresponds to separating one brane so that we are left with a massless $U(N)$ theory. It is the difference $F\left(r \rightarrow r_{0}\right)-F\left(r \gg r_{0}\right)$ that should have the interpretation of the SYM free energy, i.e

11 Note that with the choice of normalisation of $\rho$ we made for large $r$ we get $\rho \rightarrow \sqrt{2} r$. Then (4.4) is exactly the same as the sum of the two leading terms (3.1), (3.6) in the large $r$ expansion of $(2.10)$.

12 The inclusion of the $\lambda^{1 / 2}$ factor in the definition of $M$ in (4.5) may seem optional but as in (2.12) it leads to the strong-coupling expression for the free energy which does not include explicit powers of 't Hooft coupling. Similar identification of the energy scale (as the scale associated with supergravity probes) was recently suggested in [32]. If we absorb the $\lambda^{1 / 2}$ factor in $M$ then the high-temperature expansion below will contain extra $\lambda^{-n / 2}$ factors. 
which should account for the change in the number of relevant degrees of freedom. Indeed, this difference gives us $-\frac{1}{4} \pi^{2} V_{3} N T^{4}$ which happens to coincide with $F\left(r \gg r_{0}\right)$ and is exactly in agreement with order $N$ term in the (strong-coupling) SYM result (3.4).

The $T^{2} M^{2}$ and $T M^{3}$ terms are the same as the ones that appear in the standard hightemperature expansion of the perturbative (e.g., 1-loop) free energy of a massive theory (see (A.11)). However, the $T^{3} M$ term present in (4.7) is absent in the weak-coupling free energy. In general, it is natural to suggest that the large $N$, finite $\lambda$ free energy has the following high-temperature expansion (cf.(3.8))

$$
F(T \gg M)=-\frac{1}{3} \pi^{2} V_{3} N T^{4}\left[2 f(\lambda)+\kappa_{1}(\lambda) \frac{M}{T}+\kappa_{2}(\lambda) \frac{M^{2}}{T^{2}}+\kappa_{3}(\lambda) \frac{M^{3}}{T^{3}}+\ldots\right],
$$

where

$$
\kappa_{n}(\lambda \rightarrow 0) \rightarrow w_{n}, \quad \kappa_{n}(\lambda \gg 1) \rightarrow s_{n}+v_{n} \lambda^{-3 / 2}+\ldots .
$$

The weak and strong coupling coefficients $w_{n}$ and $s_{n}$ are, in general, different. In particular, $w_{1}=0$ but $s_{1} \neq 0$, while both $w_{n}$ and $s_{n}$ are non-vanishing for $n>1$.

\section{5. $\alpha^{\prime 3} R^{4}$ string correction and strong-coupling expansion of SYM free energy}

To justify our expectation about the presence of $\lambda^{-3 / 2}$ corrections to the strongcoupling expressions for the SYM free energy (3.5), (3.8), (4.8) let us now repeat the supergravity computation of section 2 now taking the non-extremal D3-brane source metric to be deformed by the leading $\alpha^{\prime 3} R^{4}$ correction to the string effective action (see [17, 10] and refs. there). As in [10] the idea is to find the changes in the probe's action and thus in the free energy. This will allow us to determine the leading correction to the strong coupling SYM result as predicted by string-corrected supergravity. Remarkably, we will find that the ' $N^{2}+2 N+1$ ' pattern for the coefficient of the leading $T^{4}$ term in the action (3.4) Survives the correction, implying that the order $N$ ('massive') contribution should be multiplied by the same function (3.3) of the 't Hooft coupling as found in the massless phase in [10].

Let us first summarize the result for the corrected near-horizon (or large $N$, large $N g_{s}$ ) limit 10-d Einstein-frame metric [10,33]:

$$
d s_{10 E}^{2}=e^{-\frac{10}{3} \nu(x)} g_{5 m n}(x) d x^{m} d x^{n}+e^{2 \nu(x)} L^{2} d \Omega_{5}^{2} .
$$

Here the 5-d metric is 10 (cf.(2.1))

$$
g_{5 m n}(x) d x^{m} d x^{n}=L^{-2} r^{2}\left(-\mathrm{ff}_{1} d t^{2}+d y_{i} d y_{i}\right)+\mathrm{f}^{-1} \mathrm{f}_{2} L^{2} r^{-2} d r^{2},
$$




$$
\begin{gathered}
\mathrm{f}=1-\left(\frac{r_{0}}{r}\right)^{4}, \quad \mathrm{f}_{1}=1-15 \gamma\left[5\left(\frac{r_{0}}{r}\right)^{4}+5\left(\frac{r_{0}}{r}\right)^{8}-3\left(\frac{r_{0}}{r}\right)^{12}\right]+O\left(\gamma^{2}\right), \\
\mathrm{f}_{2}=1+15 \gamma\left[5\left(\frac{r_{0}}{r}\right)^{4}+5\left(\frac{r_{0}}{r}\right)^{8}-19\left(\frac{r_{0}}{r}\right)^{12}\right]+O\left(\gamma^{2}\right),
\end{gathered}
$$

and the ' $S^{5}$ raduis' function is 33

$$
e^{\nu}=1+\frac{15}{32} \gamma\left[\left(\frac{r_{0}}{r}\right)^{8}+\left(\frac{r_{0}}{r}\right)^{12}\right]+O\left(\gamma^{2}\right) .
$$

Note that $\nu$ does not have $\left(\frac{r_{0}}{r}\right)^{4}$ term as this function corresponds to a massive (fixed) scalar [10]. For completeness, the expression for the 10-d dilaton which is needed to determine the 10-d string-frame metric $d s_{10}^{2}=e^{-\frac{1}{2} \phi} d s_{10 E}^{2}$ is [10]

$$
e^{\phi}=1-\frac{15}{16} \gamma\left[6\left(\frac{r_{0}}{r}\right)^{4}+3\left(\frac{r_{0}}{r}\right)^{8}+2\left(\frac{r_{0}}{r}\right)^{12}\right]+O\left(\gamma^{2}\right) \text {. }
$$

Here $\gamma$ is proportional to the coefficient of the $R^{4}$ term in the type II superstring effective action (compared to [10] we make $\gamma$ dimensionless by multiplying it by $L^{-6}$, cf.(2.4))

$$
\gamma \equiv \frac{1}{8} \zeta(3) \alpha^{\prime 3} L^{-6}=\frac{1}{8} \zeta(3) \lambda^{-3 / 2}
$$

To find the static potential term in the action of a D3-brane probe propagating in this background we also need the 'electric' part of the 4-form potential. The corresponding selfdual 5-form field strength is determined by the 5-part of the above 10-d Einstein-frame metric (5.1) (note that $F_{5}^{2}$ term is Weyl-invariant in 10 dimensions):

$$
F_{0123 r}=\partial_{r} C_{0123}=4 L^{-1} \sqrt{-h_{5}}, \quad h_{5 m n} \equiv e^{-\frac{10}{3} \nu} g_{5 m n} .
$$

Since $\sqrt{-h_{5}}=L^{-3} r^{3} e^{-\frac{25}{3} \nu} \sqrt{\mathrm{f}_{1} \mathrm{f}_{2}}$ we get

$$
C_{0123}=L^{-4} r^{4} C(r), \quad C(r)=1+\gamma\left[\frac{250}{32}\left(\frac{r_{0}}{r}\right)^{8}+\frac{3965}{64}\left(\frac{r_{0}}{r}\right)^{12}\right]+O\left(\gamma^{2}\right) .
$$

We shall assume that there are no direct $\alpha^{\prime}$-corrections to the form of the D3-brane probe action, i.e. that only the background is deformed by $O(\gamma)$ terms. Then the static potential part of the action $I=T^{-1} F$ or the free energy $F$ is (cf. $(2.10)$ 13

$$
F=T_{3} V_{3} L^{-4} r^{4}\left[\sqrt{e^{-\frac{40}{3} \nu(r)} \mathrm{f}(r) \mathrm{f}_{1}(r)}-C(r)\right]
$$

13 Note that in terms of the string-frame 10-d metric $I=T_{3} \int d^{4} x\left(e^{-\phi} \sqrt{-\operatorname{det}_{4} G_{10}}-C_{0123}\right)$ but the dilaton factor drops out once we transform to the Einstein-frame metric. 


$$
\begin{aligned}
=T_{3} V_{3} L^{-4} r^{4} & \left\{\sqrt{1-\left(\frac{r_{0}}{r}\right)^{4}}\left(1-\gamma\left[\frac{75}{2}\left(\frac{r_{0}}{r}\right)^{4}+\frac{325}{8}\left(\frac{r_{0}}{r}\right)^{8}-\frac{155}{8}\left(\frac{r_{0}}{r}\right)^{12}\right]\right)\right. \\
& \left.-1-\gamma\left[\frac{125}{32}\left(\frac{r_{0}}{r}\right)^{8}+\frac{3965}{64}\left(\frac{r_{0}}{r}\right)^{12}\right]+O\left(\gamma^{2}\right)\right\} .
\end{aligned}
$$

The leading $O(\gamma)$ correction to the strong-coupling result for the SYM free energy in the 'massless' phase (3.4) found in [10] is (see also (3.2),(3.3))

$$
F=-\frac{1}{8 \pi^{2}} N^{2} V_{3} L^{-8} r_{0}^{4}(1+75 \gamma)=-\frac{1}{8} N^{2} V_{3} T^{4}(1+15 \gamma)
$$

where we used the modified relation between the temperature and $r_{0}$ [10]

$$
T=\frac{r_{0}}{\pi L^{2}}(1+15 \gamma)
$$

In the present 'separated' case the leading $r$-independent term in the interaction potential (5.9) is found to be (cf. (3.1) 14

$$
F=-\frac{1}{2 \pi^{2}} T_{3} N V_{3} L^{-8} r_{0}^{4}\left[1+75 \gamma+O\left(\gamma^{2}\right)\right]=-\frac{1}{4} N V_{3} T^{4}\left[1+15 \gamma+O\left(\gamma^{2}\right)\right] .
$$

Up to the coefficient $2 N$ instead of $N^{2}$ (cf. (3.4) ) this is exactly the same expression as in the coinciding 3 -brane case (5.10). This is perfectly consistent with the expectation that the functions $f$ and $\tilde{f}$ in (3.5) should be the same, i.e. that the $U(N+1)$ expression (3.2) for the total free energy should apply to both coinciding and separated brane cases.

Now we can look at the subleading term in the large distance expansion of (5.9). We find the following $O(\gamma)$ correction to (3.6) (see (5.11) )

$$
\begin{gathered}
F_{1}=-\frac{1}{8}\left(1+\frac{1425}{4} \gamma+\ldots\right) V_{3} T_{3} L^{-4} \frac{r_{0}^{8}}{r^{4}} \\
=-\frac{1}{16} \pi^{6} V_{3} N \lambda^{2}\left[1+\frac{945}{32} \zeta(3) \lambda^{-3 / 2}+\ldots\right] \frac{T^{8}}{U^{4}} .
\end{gathered}
$$

This determines the first two terms in the function $h_{1}(\lambda)$ in (3.8).

Similar analysis can be repeated in the case of the near-horizon $r \rightarrow r_{0}$ expansion of the interaction potential discussed in section 4. As follows from (5.9), the leading $r_{0}^{4}$ term in (4.4) gets multiplied by $1+\frac{4265}{64} \gamma$. To find the modification of the subleading terms one has first to put the 6-d part of the metric (5.1) in the 'isotropic' form as in (4.2), thus obtaining the $O(\gamma)$ correction to the relation (4.3) between $r \simeq r_{0}$ and the new coordinate $\rho$. Then one needs to express the action (5.9) in terms of $\rho$. Instead of (4.3) we now get $\rho=\exp \int \frac{d r}{r \sqrt{\mathrm{f}}} \sqrt{\mathrm{f}_{2}} e^{-\frac{8}{3} \nu}=\exp \int \frac{d r}{r \sqrt{1-\left(\frac{r_{0}}{r}\right)^{4}}}\left(1+\frac{1}{4} \gamma\left[150\left(\frac{r_{0}}{r}\right)^{4}+145\left(\frac{r_{0}}{r}\right)^{8}-575\left(\frac{r_{0}}{r}\right)^{12}\right]\right)$.

The final effect of expressing the action in terms of $\rho$ and expanding in powers of $\frac{M}{T}$ can be seen by using the leading-order relations (4.3), (4.5) in the $O(\gamma)$ terms in (5.9): we get $1+c_{n} \gamma$ factors for all $\left(\frac{M}{T}\right)^{n}$ terms in the expansion of the free energy, confirming that $F$ has the general structure suggested in $(4.8),(4.9)$.

14 Note that the non-trivial dependence of $\nu$ on $r$ is irrelevant to this order as (5.4) starts with the $\left(\frac{r_{0}}{r}\right)^{8}$ term. 


\section{Acknowledgements}

We are grateful to O. Aharony, N. Itzhaki, I. Klebanov and M. Peskin for useful and stimulating discussions. A.T. acknowledges the High Energy Theory Group of Rutgers University for hospitality during the completion of this work. S.Y. would like to thank the theory group at SLAC for its hospitality. The work of A.T. is supported in part by PPARC, the European Commission TMR programme grant ERBFMRX-CT96-0045 and the INTAS grant No.96-538. The work of S.Y. is supported in part by the US-Israel Binational Science Foundation, by GIF - the German-Israeli Foundation for Scientific Research, and by the Israel Science Foundation. 


\section{Appendix A. One-loop $\mathcal{N}=4 \mathrm{SYM}$ finite temperature effective potential}

In general, the 1-loop free energy of a gas of $N_{B}$ scalars and $N_{F}$ spinors of the same mass $M$ has the following high temperature $(T \gg M)$ expansion (see e.g. [34)

$$
\begin{aligned}
F=- & V_{3}\left[\frac{\pi^{2}}{90}\left(N_{B}+\frac{7}{8} N_{F}\right) T^{4}-\frac{1}{24}\left(N_{B}+\frac{1}{2} N_{F}\right) M^{2} T^{2}+\frac{1}{12 \pi} N_{B} M^{3} T\right. \\
& \left.+\frac{1}{32 \pi^{2}}\left(N_{B}-N_{F}\right) M^{4} \ln \frac{M}{\pi T}-\frac{1}{16 \pi^{2}} N_{B} M^{4} \ln 2+\ldots\right] .
\end{aligned}
$$

The supersymmetric case corresponds to $N_{B}=N_{F}$. In the case of $\mathcal{N}=4 U(N) \mathrm{SYM}$ theory in the vacuum with zero scalar values one has $N_{B}=N_{F}=8 N^{2}$. In the vacuum with the scalar value $U$ breaking $U(N+1)$ to $U(N) \times U(1)$ the contribution of the massive $(M \sim U)$ states to the free energy is determined by (A.1) with $N_{B}=N_{F}=16 N$.

In a general $3+1$ dimensional field theory with bosonic and fermionic degrees of freedom with mass operators $\hat{M}_{B}$ and $\hat{M}_{F}$ (which may depend on background fields) the proper-time representation for the one-loop free energy has the form

$$
F=-\frac{V_{3}}{2(4 \pi)^{2}} \int_{0}^{\infty} \frac{d \mathrm{t}}{\mathrm{t}^{3}}\left[\theta_{3}\left(0 \mid \frac{i \beta^{2}}{4 \pi \mathrm{t}}\right) \operatorname{Tr} e^{-\mathrm{t} \hat{M}_{B}^{2}}-\theta_{4}\left(0 \mid \frac{i \beta^{2}}{4 \pi \mathrm{t}}\right) \operatorname{Tr} e^{-\mathrm{t} \hat{M}_{F}^{2}}\right] .
$$

The inverse temperature $\beta=T^{-1}$ is the period of the euclidean time direction. Using the relation for the Jacobi $\theta$-functions

$$
\theta_{3}(0 \mid i z) \pm \theta_{4}(0 \mid i z)=\sum_{n=-\infty}^{\infty}\left[1 \pm(-1)^{n}\right] e^{-\pi z n^{2}}=2 \theta_{3,2}(0 \mid 4 i z)
$$

in the special case of a free supersymmetric theory with equal bosonic and fermionic masses $\hat{M}_{B}=\hat{M}_{F}=\hat{M}$ this becomes (see e.g. [13])

$$
F=-\frac{V_{3}}{(4 \pi)^{2}} \int_{0}^{\infty} \frac{d \mathrm{t}}{\mathrm{t}^{3}} \theta_{2}\left(0 \mid \frac{i \beta^{2}}{\pi \mathrm{t}}\right) \operatorname{Tr} e^{-\mathrm{t} \hat{M}^{2}}=-\frac{V_{3} T^{4}}{(4 \pi)^{2}} \int_{0}^{\infty} \frac{d t}{t^{3}} \theta_{2}\left(0 \mid \frac{i}{\pi t}\right) \operatorname{Tr} e^{-t \frac{\hat{\hat{N}}^{2}}{T^{2}}} .
$$

If the fields are massless with total $N_{B}$ of bosonic degrees of freedom $\left(N_{B}=8 N^{2}\right.$ for $U(N)$ $\mathcal{N}=4 \mathrm{SYM})$

$$
F=-\frac{\pi^{2}}{6} N_{B} V_{3} T^{4}
$$

At zero temperature the 1-loop effective action of $\mathcal{N}=4$ SYM theory in a general homogeneous scalar background has the following form [35]

$$
S_{e f f}=-\frac{V_{4}}{2(4 \pi)^{2}} \int \frac{d \mathrm{t}}{\mathrm{t}^{3}} \operatorname{Tr}\left(e^{-\mathrm{t} \hat{M}_{1}^{2}}+2 e^{-\mathrm{t} \hat{M}^{2}}-\frac{1}{4} e^{-\mathrm{t} \hat{M}_{1 / 2}^{2}}\right)
$$


where the mass matrices are

$$
\begin{gathered}
\left(\hat{M}^{2}\right)^{a b}=f_{\text {aec }} f_{b e d} A_{i}^{c} A_{i}^{d}, \quad\left(\hat{M}_{1}^{2}\right)_{i j}^{a b}=\delta_{i j}\left(\hat{M}^{2}\right)^{a b}-2 F_{i j}^{a b}, \\
\left(\hat{M}_{1 / 2}^{2}\right)_{\alpha \beta}^{a b}=\delta_{\alpha \beta}\left(\hat{M}^{2}\right)^{a b}-\frac{1}{2}\left(\gamma_{i j}\right)_{\alpha \beta} F_{i j}^{a b} .
\end{gathered}
$$

Here $F_{i j}^{a b}=\left[A_{i}, A_{j}\right]^{a b}=f^{a c b} f^{c e d} A_{i}^{e} A_{j}^{d}, \quad i=1, \ldots, 6$ are the internal 6 -space indices, $a, b, \ldots$ are the group indices, and $\alpha, \beta=1, \ldots, 32$ are the $10-\mathrm{d}$ spinor indices. Traces are taken in respective spaces, e.g., for the unit operators $\operatorname{Tr}\left(I_{1}+2 I_{0}-\frac{1}{4} I_{1 / 2}\right)=N^{2}\left(6+2-\frac{1}{4} \cdot 32\right)=0$. The expression (A.6) is obtained [35] by using that the $\mathcal{N}=4 \mathrm{SYM}$ is a dimensional reduction of the $D=10 \mathrm{SYM}$ theory. In particular, the scalar matrix $A_{i}^{a}$ is just the internal component of the $10-\mathrm{d}$ vector potential. Note that the mass matrix does not contain $g_{\mathrm{YM}}^{2}$ factor $\left(g_{\mathrm{YM}}^{-2}\right.$ appears in front of the whole action).

In the case of a vacuum (Cartan direction) scalar condensate (corresponding to separated parallel D3-brane configuration) one has $F_{i j}^{a b}=0$ so that all mass matrices coincide (reflecting the remaining supersymmetry). UV divergences cancel as a result of the $\mathcal{N}=4$ sum rules:

$$
\operatorname{Tr}\left[\left(\hat{M}_{1}^{2}\right)^{k}+2\left(\hat{M}^{2}\right)^{k}-\frac{1}{4}\left(\hat{M}^{2}\right)^{k}\right]=0, \quad k=0, \ldots, 3 .
$$

To obtain the finite temperature analogue of (A.6) one is simply to add the thermal $\theta$ function factors in (A.2) inside the integral in (A.6) and to use that $V_{4}=V_{3} \beta$

$$
F=-\frac{V_{3}}{2(4 \pi)^{2}} \int_{0}^{\infty} \frac{d \mathrm{t}}{\mathrm{t}^{3}}\left[\theta_{3}\left(0 \mid \frac{i \beta^{2}}{4 \pi \mathrm{t}}\right) \operatorname{Tr}\left(e^{-\mathrm{t} \hat{M}_{1}^{2}}+2 e^{-\mathrm{t} \hat{M}^{2}}\right)-\frac{1}{4} \theta_{4}\left(0 \mid \frac{i \beta^{2}}{4 \pi \mathrm{t}}\right) \operatorname{Tr} e^{-\mathrm{t} \hat{M}_{1 / 2}^{2}}\right]
$$

Assuming $F_{i j}=0$ and doing the traces over $S O(6)$ and spinor indices we get (cf.(A.4))

$$
F=-\frac{8 V_{3}}{2(4 \pi)^{2}} \int_{0}^{\infty} \frac{d \mathrm{t}}{\mathrm{t}^{3}} \theta_{2}\left(0 \mid \frac{i \beta^{2}}{\pi \mathrm{t}}\right) \operatorname{Tr} e^{-\mathrm{t} \hat{M}^{2}}
$$

where the remaining trace is over the group indices. We shall consider the case of a simple $U(N+1) \rightarrow U(N) \times U(1)$ breaking (a single brane separated from $N$ others) when the matrix $\hat{M}^{2}$ has only one non-zero $2 N$-degenerate eigenvalue $M^{2}$. Then $F$ is given by the sum of the massless and massive contributions

$$
F=F_{0}+F_{M}=-\frac{8 V_{3}}{2(4 \pi)^{2}} \int_{0}^{\infty} \frac{d \mathrm{t}}{\mathrm{t}^{3}} \theta_{2}\left(0 \mid \frac{i \beta^{2}}{\pi \mathrm{t}}\right)\left[\left(N^{2}+1\right)+2 N e^{-\mathrm{t} M^{2}}\right] .
$$

To expand the massive contribution at large $T \gg M$ one may rescale $\mathrm{t}$ so that

$$
F_{M}=-\frac{16 N V_{3} T^{4}}{2(4 \pi)^{2}} \int_{0}^{\infty} \frac{d t}{t^{3}} \theta_{2}\left(0 \mid \frac{i}{\pi t}\right) e^{-t \frac{M^{2}}{T^{2}}}
$$


getting

$$
F_{M}(T \gg M)=-\frac{\pi^{2}}{6} 2 N V_{3}\left(T^{4}-3 \pi^{-2} T^{2} M^{2}+4 \pi^{-3} T M^{3}+\ldots\right) .
$$

For small $T \ll M$ we use that $\theta_{2}\left(0 \mid \frac{i \beta^{2}}{\pi \mathrm{t}}\right)_{\beta \rightarrow \infty} \rightarrow 2 e^{-\frac{\pi \beta^{2}}{4 \mathrm{t}}}$, and the resulting integral is proportional to

$$
\int_{0}^{\infty} d \mathrm{t} \mathrm{t}^{-3} \exp \left(-\mathrm{t} M^{2}-\frac{\pi \beta^{2}}{4 \mathrm{t}}\right)=16 \frac{M^{2}}{\pi \beta^{2}} K_{2}(\sqrt{\pi} \beta M) .
$$

It may be evaluated, e.g., by the saddle-point method. Instead of having power-series expansion in $\frac{M}{T}$, for $M \gg T$ the free energy thus goes to zero exponentially as $V_{3} M^{2} T^{2} \exp \left(-\sqrt{\pi} \frac{M}{T}\right)$. 


\section{References}

[1] J. Polchinski, Dirichlet Branes and Ramond-Ramond charges, Phys. Rev. Lett. 75 (1995) 4724, hep-th/9510017.

[2] M.R. Douglas, D. Kabat, P. Pouliot and S.H. Shenker, Nucl. Phys. B485 (1997) 85, hep-th/9608024.

[3] S.S. Gubser, I.R. Klebanov and A. Peet, Entropy and temperature of black 3-branes, Phys. Rev. D54 (1996) 3915, hep-th/9602135.

[4] I.R. Klebanov, World volume approach to absorption by nondilatonic branes, Nucl. Phys. B496 (1997) 231, hep-th/9702076.

[5] S.S. Gubser, I.R. Klebanov and A.A. Tseytlin, String theory and classical absorption by three-branes, Nucl. Phys. B499 (1997) 217, hep-th/9703040.

[6] M. Douglas, J. Polchinski and A. Strominger, Probing five-dimensional black holes with D-branes, JHEP 12 (1997) 003, hep-th/9703031.

[7] J. Maldacena, The Large N limit of superconformal field theories and supergravity, Adv. Theor. Math. Phys. 2 (1998) 231, hep-th/9711200.

[8] S.S. Gubser, I.R. Klebanov and A.M. Polyakov, Gauge theory correlators from noncritical string theory, Phys. Lett. B428 (1998) 105, hep-th/9802109.

[9] E. Witten, Anti-de Sitter space and holography, Adv. Theor. Math. Phys. 2 (1998) 253, hep-th/9802150.

[10] S.S. Gubser, I.R. Klebanov and A.A. Tseytlin, Coupling constant dependence in the thermodynamics of N=4 supersymmetric Yang-Mills theory, Nucl. Phys. B534 (1998) 202, hep-th/9805156.

[11] J. Maldacena, Probing near-extremal black holes with D-branes, Phys. Rev. D57 (1998) 3736, hep-th/9705053.

[12] J. Maldacena, Branes probing black holes, Nucl. Phys. Proc. Suppl. 68 (1998) 17, hep-th/9709099.

[13] A.A. Tseytlin, Open superstring partition function in constant gauge field background at finite temperature, Nucl. Phys. B524 (1998) 41, hep-th/9802133.

[14] G.T. Horowitz and A. Strominger, Black strings and p-branes, Nucl. Phys. B360 (1991) 197.

[15] M.J. Duff and J.X. Lu, The selfdual type IIB superthreebrane, Phys. Lett. B273 (1991) 409.

[16] D.A. Kirzhnits and A.D. Linde, Phys. Lett. B42 (1972) 471; S. Weinberg, Phys. Rev. D9 (1974) 2257; L. Dolan and R. Jackiw, Phys. Rev. D9 (1974) 3320.

[17] T. Banks and M.B. Green, Non-perturbative effects in $A d S_{5} \times S^{5}$ string theory and $d=4$ susy Yang-Mills, J.High Energy Phys.05 (1998) 002, hep-th/9804170.

[18] M. Li, Evidence for large $N$ phase transition in $\mathcal{N}=4$ super Yang-Mills theory at finite temperature, hep-th/9807196. 
[19] I.R. Klebanov and A.A. Tseytlin, Entropy of near-extremal black p-branes, Nucl. Phys. B475 (1996) 164, hep-th/9604089.

[20] G.T. Horowitz and S.F. Ross, Possible resolution of black hole singularities from large $N$ gauge theory, JHEP 04 (1998) 015, hep-th/9803085.

[21] J. Kapusta, D. Reiss and S. Rudas, Transition from weak to strong coupling in QCD and in grand unified models at high temperature, Nucl. Phys. B263 (1986) 207.

[22] E. Witten, Anti de Sitter space, thermal phase transition, and confinement in gauge theories, Adv. Theor. Math. Phys. 2 (1998) 505, hep-th/9803131.

[23] A. Brandhuber, N. Itzhaki, J. Sonnenschein and S. Yankielowicz, Wilson loops in the large $N$ limit at finite temperature, hep-th/9803137.

[24] S.-J. Rey, S. Theisen and J.-T. Yee, Wilson-Polyakov loop at finite temperature in large $N$ gauge theory, Nucl. Phys. B527 (1998) 171, hep-th/9803135.

[25] M.R. Douglas and M. Li, D-brane realization of $N=2$ super Yang-Mills theory in four dimensions, hep-th/9604041; M.R. Douglas and W. Taylor, Branes in the bulk of Anti de Sitter space, hep-th/9807225.

[26] T. Banks, W. Fischler, S.H. Shenker and L. Susskind, M theory as a matrix model: a conjecture, Phys. Rev. D55 (1997) 5112, hep-th/9610043.

[27] K. Becker, M. Becker, J. Polchinski and A.A. Tseytlin, Higher order graviton scattering in M(atrix) theory, Phys. Rev. D56 (1997) 3174, hep-th/9706072.

[28] I. Chepelev and A.A. Tseytlin, Long distance interactions of branes: Correspondence between supergravity and superYang-Mills descriptions, Nucl. Phys. B515 (1998) 73, hep-th/9709087.

[29] M. Dine and N. Seiberg, Comments on higher derivative operators in some susy field theories, Phys. Lett. B409 (1997) 239, hep-th/9705057; S. Paban, S. Sethi and M. Stern, Supersymmetry and higher derivative terms in the effective action of YangMills theories, JHEP 06 (1998) 012, hep-th/9806028.

[30] S.S. Gubser and I.R. Klebanov, Absorption by Branes and Schwinger Terms in the World Volume Theory, Phys. Lett. B413 (1997) 41, hep-th/9708005.

[31] X. Kong and F. Ravndal, Quantum Corrections to the QED Vacuum Energy, Nucl. Phys. B526 (1998) 627, hep-ph/9803216.

[32] A.W. Peet and J. Polchinski, UV/IR relations in AdS dynamics, hep-th/9809022.

[33] J. Pawelczyk and S. Theisen, $A d S_{5} \times S^{5}$ Black Hole Metric at $O\left(\alpha^{\prime 3}\right)$, hep-th/9808126.

[34] J.I. Kapusta, Finite-temperature field theory (Cambridge Univ. Press, 1989).

[35] E.S. Fradkin and A.A. Tseytlin, Quantum properties of higher dimensional and dimensionally reduced supersymmetric theories, Nucl. Phys. B227 (1983) 252. 